\title{
Fluralaner 5.46\% (W/W) Flavored Chewable Tablet (Bravecto@ 1-Month) Is Effective for Treatment of Canine Generalized Demodicosis
}

Nadja Rohdich ( $\square$ nadja.rohdich@msd.de)

MSD Animal Health Innovation $\mathrm{GmbH}$

Leon Meyer

Clinvet International

Frank Guerino

Merck Animal Health

\section{Research}

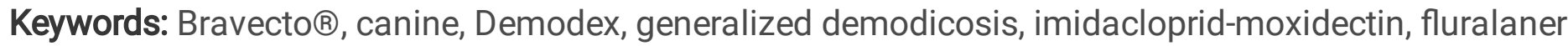

Posted Date: October 5th, 2021

DOl: https://doi.org/10.21203/rs.3.rs-948420/v1

License: (c) (i) This work is licensed under a Creative Commons Attribution 4.0 International License.

Read Full License

Version of Record: A version of this preprint was published at Parasites \&amp; Vectors on March 12th, 2022. See the published version at https://doi.org/10.1186/s13071-022-05213-x. 


\section{Abstract}

Background: Orally administered fluralaner $(13.64 \% \mathrm{w} / \mathrm{w})$ is effective for treating canine generalized demodicosis. A study was initiated to assess the efficacy of a novel $5.46 \% \mathrm{w} / \mathrm{w}$ fluralaner chewable tablet formulation for monthly administration in the treatment of this disease.

Methods: Client-owned dogs diagnosed with generalized demodicosis were acclimatized to laboratory conditions and randomized to receive either orally administered fluralaner (Bravecto ${ }^{\circledR}$ 1-Month) (10.0 to $14.4 \mathrm{mg} / \mathrm{kg}$ body weight) $\left(\mathrm{n}=8\right.$ ) or topical imidacloprid-moxidectin (Advocate ${ }^{\circledR}$ for dogs, Elanco) applied per label on Days 0,28 and $56(n=8)$, or more frequently for ongoing severe demodicosis. On Days $-2,28$, 56 and 84, deep skin scrapings were taken from 5 sites on each dog for mite identification and counting, and semi-quantitative clinical assessments of generalized demodicosis were recorded. Primary efficacy was based upon arithmetic mean mite count reductions relative to pre-treatment.

Results: By Day 28 , mean pre-treatment mite counts, $>600$ in both groups, were significantly reduced by $99.7 \%$ and $89.5 \%$ (both $P<0.001$ ) in the fluralaner and imidacloprid-moxidectin groups, respectively. Parasitological cure (100\% reduction in mite counts on Days 56 and 84$)$ was achieved in all fluralanertreated dogs (100\%) and in 2 imidacloprid-moxidectin treated dogs (25\%). In the imidacloprid-moxidectin group, the reduction in mean mite counts was $89.5 \%$ (Day 28), $94.4 \%$ (Day 56) and $97.5 \%$ (Day 84). All study dogs were free of crusts on Days 56 and 84. Scales resolved by Day 84 in all fluralaner-treated dogs and in 3 imidacloprid-moxidectin treated dogs. All fluralaner-treated dogs and 5 imidaclopridmoxidectin treated dogs had $>90 \%$ hair re-growth on Day 84 .

Conclusion Three consecutive monthly oral administrations of fluralaner $(5.46 \% \mathrm{w} / \mathrm{w})$ flavored chewable tablets (minimum dose rate $10 \mathrm{mg} / \mathrm{kg}$ body weight) eliminated Demodex canis mites from dogs diagnosed with generalized demodicosis.

\section{Background}

Canine demodicosis is an inflammatory parasitic skin disease characterized by an excessive multiplication of Demodex canis mites within the pilosebaceous glands [1]. Localized demodicosis is generally a benign disease with most cases resolving spontaneously within 6 to 8 weeks of the appearance of clinical signs [2]. The disease is defined as generalized when 5 or more areas of the body are affected, pododemodicosis is observed on at least 2 paws, or when an entire body region is involved. Demodicosis is also categorized as either juvenile (dogs up to 18 months of age) or adult onset (dogs generally older than four years of age with no previous history of disease), or chronic generalized (disease persisting for at least six months) [2]. Generalized demodicosis is unlikely to resolve without therapy and has long provided a challenge to veterinarians to implement successful treatment [2 - 4). Until recently, a topically applied combination formulation of imidacloprid and moxidectin was the only registered product for the treatment of canine generalized demodicosis. However, even with weekly re- 
treatments, efficacy has been shown to be incomplete $[5,6]$. The discovery and registration of the isoxazoline family of compounds provided a breakthrough in treatment of this complex disease.

The isoxazoline compound fluralaner was first available in 2014 as a flavored chewable tablet formulation to provide up to 12 weeks efficacy against canine flea and common tick infestations, and is now also approved for the treatment of sarcoptic and demodectic mange infestations [7]. In 2016 a topical formulation of fluralaner was approved for use in cats as well as dogs to provide the same efficacy and duration as the original oral formulation for the treatment and control of flea, tick and mite infestations. More recently, a novel $5.46 \%(\mathrm{w} / \mathrm{w})$ fluralaner chewable tablet formulation was developed for monthly administration at a minimum dose rate of $10 \mathrm{mg} / \mathrm{kg}$ to control canine flea and tick infestations [8]. This study was conducted to evaluate the efficacy of the new tablet formulation in the treatment of canine generalized demodicosis when administered three times at monthly intervals.

\section{Methods}

This study was conducted in accordance with the VICH GL9 (June 2000) guideline on Good Clinical Practice [9], and Guideline on Statistical Principles for Veterinary Clinical Trials [10]. Ethical approval was obtained by the ClinVet Institutional Animal Care and Use Committee before beginning the study, and all owners signed informed consent forms after receiving written and verbal details of the study procedures and products to be administered.

\section{Dogs and Management}

To qualify for the study, dogs were required to be clinically healthy, except for clinical signs associated with generalized demodicosis; to present with clinical signs of infestation (including erythema, hair loss, comedones, follicular casts, scales and crusts) affecting more than 5 sites on the body, pododemodicosis involving at least 2 paws, or an entire body region). Dogs were excluded if they had received glucocorticoid therapy or any ectoparasiticide or endectocide within 12 weeks prior to Day 0 , if known to be pregnant, or if fractious to the point of posing a danger to themselves or facility personnel.

Adult dogs were sourced from rural areas with limited access to animal health care. Following owner consent, 16 suitable dogs were transferred to the study site to undergo a 7-day acclimatization period, during which daily health observations were made, with clinical examinations and body weight measurements completed on Days -7 and -2. Starting on Day -7 all dogs received an appropriate antibiotic (Convenia ${ }^{\circledR}$, cefovecin, Zoetis) as a treatment for pyoderma. The dogs also received a probiotic (Protexin ${ }^{\circledR}$ Soluble, Kyron Laboratories) from Day -7 until the end of the study on Day 84 . Demodicosis was confirmed by identification of $D$. canis mites from deep skin scrapings completed on Day -2 .

During the study, dogs were kept individually in concrete-floored pens under strict quarantine conditions. No physical contact between dogs was possible, but dogs had visual and auditory contact with conspecifics. The dog pens consisted of a $1.69 \mathrm{~m} \times 0.7 \mathrm{~m}$ enclosed sleeping area, with panel heaters, 
plastic beds/rubber mats and an outside run of $1.69 \mathrm{~m} \times 3.0 \mathrm{~m}$. Standard commercially available diets that met the nutritional requirements of the dogs were fed at the recommended rates, and water was provided in stainless steel bowls and replenished at least twice daily. Food was removed in the afternoon prior to skin biopsies to facilitate anesthesia for biopsy procedures. At least one toy/chew was available to each dog (replenished weekly). A roof covering the kennels prevented exposure to rain and dogs were held in ambient temperatures with natural lighting.

\section{Study Treatments}

On treatment days, dogs consumed up to half of their daily food ration within approximately 20 minutes before treatment, after which they were offered the remainder of the ration so that all were in the fed state at the time of treatment. Dogs were ranked according to live mite counts and randomized to a treatment group. Dogs allocated to Group 1 were treated with a new formulation of fluralaner $(5.46 \% \mathrm{w} / \mathrm{w}$ flavored chewable tablets) (Bravecto ${ }^{\circledR} 1$-Month) on Days 0,28 and 56 at a minimum dose rate of $10 \mathrm{mg} / \mathrm{kg}$ (actual dose rates 10.0 to $14.4 \mathrm{mg} / \mathrm{kg}$ ). Group 2 dogs were treated with a commercially available topical formulation containing imidacloprid $(100 \mathrm{mg} / \mathrm{mL})$ and moxidectin $(25 \mathrm{mg} / \mathrm{mL})$ (Advocate $\left.{ }^{\circledR}\right)$ administered according to label directions ( $10 \mathrm{mg}$ imidacloprid $/ \mathrm{kg}, 2.5 \mathrm{mg}$ moxidectin $/ \mathrm{kg}$ ). Treatments with imidacloprid-moxidectin were applied directly to the skin at one spot between the shoulder blades. Dogs were restrained for approximately one minute following topical administration and any drip-off was reapplied. Although scheduled for applications on Days 0,28 and 56, the frequency of treatments with this topical product could be increased to once weekly if the blinded attending veterinarian diagnosed the demodicosis as being severe. Appropriate dosing for study treatments was calculated according to each dog's body weight, assessed on Days -2, 27 and 56. On Day -2 weights of dogs in Group 1 ranged from 9.7 to $17.6 \mathrm{~kg}$ (median $13.0 \mathrm{~kg}$ ) and in Group 2 from 7.1 to $16.4 \mathrm{~kg}$ (median $9.0 \mathrm{~kg}$ ). To prevent unmasking during assessments, all dogs in the fluralaner group received saline solution topically during the treatment process. If any dogs in the imidacloprid-moxidectin group required weekly treatment, all dogs in both treatment groups, excluding those receiving the active topical product, were treated topically with saline solution on the same days to maintain masking of study personnel. All dogs were observed hourly for up to 4 hours after treatment and full clinical examinations were completed at 2-week intervals from Days 14 to 84 . No additional treatments with potential miticidal activity were used during the study on the dogs or their environment.

\section{Mite counts, clinical assessments, and skin biopsies}

On Days $-2,28,56$ and 84, deep scrapings were taken from 5 sites on each study dog using a scalpel blade to a skin depth at which capillary blood began oozing. Subsequent deep scrapings were made from the same sites or from sites of new lesions. Each scraping was transferred to a uniquely identified microscope slide containing mineral oil and examined under a stereo microscope to identify and count live $D$. canis mites.

Clinical signs and the extent of demodectic lesions on each dog were assessed on the days on which scrapings were made and sketched on a silhouette to assess the degree of body area covered by casts, 
scales, crusts, erythema and pyoderma, and to score body areas with hair loss ( 1 = slight thinning of hair; $2=$ conspicuous hair loss; $3=$ no hair). A semi-quantitative assessment of hair re-growth compared to the pre-treatment assessment was also completed ( $1=0$ to $50 \% ; 2=>50 \%$ to $\leq 90 \% ; 3=>90 \%)$. The overall severity of demodicosis (mild, moderate, severe) was evaluated by the blinded veterinarian investigator.

On Days -7 and 27, biopsies from affected skin areas were taken from each dog under general anesthesia (propofol 1\%). Any dogs with clinical signs of pyoderma at the Day 27 skin biopsy examination were treated with an appropriate antibiotic (Convenia ${ }^{\circledR}$ ), administered according to label. Antibiotic therapy was continued until receipt of the biopsy results, and if evidence of active infection was present the antibiotic treatment was continued in affected dogs for another four weeks. From Day 0 until the end of the study all clinical assessments and mite counts were completed by qualified personnel blinded to treatment group.

\section{Statistical analysis}

The European Committee for Medicinal Products for Veterinary Use (CVMP) guideline for evaluation of the efficacy of anti-parasitic substances for the treatment and prevention of tick and flea infestations in cats and dogs states that at least 6 animals should be used per group [11]. For this study the guideline minimum was exceeded with 2 additional dogs included in each treatment group.

Primary efficacy was based upon the percent reduction in arithmetic mean mite counts relative to pretreatment counts.

\begin{tabular}{|ll|}
\hline Reduction [\%] $=\quad 100 \times$ & Mean $_{\text {pre }}-$ Mean $_{\text {post }}$ \\
\cline { 2 - 2 } & Mean pre \\
\hline
\end{tabular}

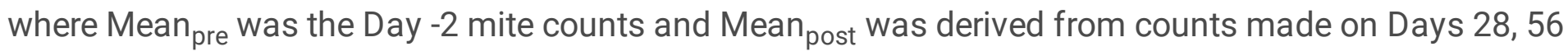
and 84 .

Geometric mean mite counts were calculated using logarithm transformed counts (count +1 ) with 1 subsequently subtracted and analyzed using a Linear Mixed Model. The fixed factor in the model was the time point. An un-adjusted comparison of the pre-treatment mite counts versus the post-treatment mite counts was performed using a two-tailed test where the level of significance was set to $a=0.05$.

The parasitological cure rate was defined as the percentage of treated dogs having two consecutive negative scrapings on Days 56 and 84. Non-inferiority and superiority of the proportion of cured fluralaner-treated dogs in comparison to the proportion of cured dogs in the imidacloprid-moxidectin group were investigated using the Farrington-Manning test of non-inferiority for the risk difference [12] with a level of significance of $a=0.025$ and a tolerated difference of $\delta=0.15$. Both $P$-value and lower $97.5 \%$ one-sided confidence limits were calculated. If the lower confidence limit was above -0.15 , noninferiority was concluded. If the lower confidence limit was above 0 , superiority was concluded. 


\section{Results}

\section{Mite counts}

All study dogs met the inclusion criteria for generalized demodicosis with mites observed in deep scrapings and accompanying clinical signs.

Arithmetic mean pre-treatment mite counts in each group exceeded 600 and by Day 28 mite counts were reduced by $99.7 \%$ in the fluralaner group and $89.5 \%$ in the imidacloprid-moxidectin group (Table $1 ;$ Fig. 1 ). Efficacy was $100 \%$ in the fluralaner group (Days 56 and 84) and in the imidacloprid-moxidectin group efficacy was $94.4 \%$ (Day 56) and $97.5 \%$ (Day 84 ). Because of the continuing severity of the clinical signs of demodicosis, dogs in the imidacloprid-moxidectin group received between 6 and 12 topical treatments, with at least a 1-week interval between consecutive treatments. One Sarcoptes scabiei mite was observed in one dog in the imidacloprid-moxidectin group prior to treatment, but no Sarcoptes spp mites were seen at any post-treatment assessment.

Table 1

Results from counts of Demodex canis mites obtained from deep skin scrapings

\begin{tabular}{|llll|}
\hline & & Fluralaner tablets & Topical imidacloprid-moxidectin \\
\hline Day -2 & Arithmetic mean (SD) & $639.9(589.9)$ & $608.5(459.5)$ \\
& Median & 507.5 & 459.0 \\
\hline & Range & $19-1764$ & $90-1314$ \\
\hline Day 28 & Arithmetic mean (SD) & $2.0(2.8)$ & $63.9(118.3)$ \\
& Median & 0.0 & 17.0 \\
\hline & Range & $0-6$ & $0-351$ \\
& P-value (vs Day -2) & $<0.001$ & $<0.001$ \\
\hline Day 56 & Arithmetic mean (SD) & $0.0(0.0)$ & $34.1(70.3)$ \\
\hline & Median & 0.0 & 11.0 \\
& Range & $0-0$ & $0-207$ \\
& P-value (vs Day -2) & $<0.001$ & $<0.001$ \\
\hline Day 84 & Arithmetic mean (SD) & $0.0(0.0)$ & $15.0(22.8)$ \\
\hline & Median & 0.0 & 3.5 \\
\hline & Range & $0-0$ & $0-65$ \\
\hline & P-value (vs Day -2) & $<0.001$ & $<0.001$ \\
\hline
\end{tabular}


At the Day 28 assessment, mites collected prior to treatment were not detected in 5 of 8 fluralaner-treated dogs (Fig. 2). All 7 fluralaner-treated dogs were negative for mites on Days 56 and 84, meeting the definition of parasitological cure (2 consecutive monthly assessments with 0 mites). Parasitological cure was recorded in 2 of the 8 imidacloprid-moxidectin-treated dogs. One imidacloprid-moxidectin treated dog received 12 weekly treatment applications, and mite counts from this dog were reduced to zero at all subsequent assessments with no recorded improvement in clinical signs. This was the only study dog with a severe demodicosis classification on Day 84 (Fig. 3). The number of parasitologically cured dogs in the fluralaner group $(100 \%)$ was significantly larger $(P<0.001)$ than the number in the imidaclopridmoxidectin group (25\%).

\section{Dermatological assessments}

All dogs in the imidacloprid-moxidectin group, and five dogs in the fluralaner group had severe generalized demodicosis pre-treatment on Day -2 (Fig. 4). All dogs in both groups were free of crusts at examination on Days 56 and 84 . Scales resolved by Day 84 in all fluralaner group dogs and in 5 of 8 imidacloprid-moxidectin group dogs. Casts, erythematous papules or pyoderma were not observed on any dog in either study group. Marked hair re-growth was observed in all study dogs at Day 84 . All fluralaner treated dogs had $>90 \%$ hair re-growth on Day 84 and 5 imidacloprid-moxidectin treated dogs had $>90 \%$ hair re-growth on Day 84 (Table 3) (Figs. 4 and 5).

Table 2

Number of dogs with skin lesions associated with generalized demodicosis

\begin{tabular}{|c|c|c|c|c|}
\hline & Day -2 & Day 28 & Day 56 & Day 84 \\
\hline & \multicolumn{4}{|c|}{ Fluralaner tablets } \\
\hline Crusts & $1 / 8(12.5 \%)$ & 1/8 (12.5\%) & $0 / 7(0.0 \%)$ & $0 / 7(0.0 \%)$ \\
\hline \multirow[t]{2}{*}{ Scales } & $5 / 8(62.5 \%)$ & 1/8 (12.5\%) & $3 / 7(42.9 \%)$ & $0 / 7(0.0 \%)$ \\
\hline & \multicolumn{4}{|c|}{ Topical imidacloprid-moxidectin } \\
\hline Crusts & $2 / 8(25.0 \%)$ & $0 / 8(0.0 \%)$ & $0 / 8(0.0 \%)$ & $0 / 8(0.0 \%)$ \\
\hline Scales & $7 / 8(87.5 \%)$ & $5 / 8(62.5 \%)$ & $6 / 8(75 \%)$ & $3 / 8(37.5 \%)$ \\
\hline
\end{tabular}


Table 3

Assessment of hair re-growth on dogs before and after treatment for generalized demodicosis

\begin{tabular}{lllll}
$\begin{array}{l}\text { Day of } \\
\text { Study }\end{array}$ & Score & $\begin{array}{l}\text { Body areas with hair re-growth versus pre- } \\
\text { administration assessment }\end{array}$ & $\begin{array}{l}\text { Fluralaner } \\
\text { tablets }\end{array}$ & $\begin{array}{l}\text { Topical } \\
\text { imidacloprid } \\
\text { moxidectin }\end{array}$ \\
\hline & Assessment prior to any study treatments & \\
\hline-2 & 1 & Alopecia $>0$ to $50 \%$ of the body & $\begin{array}{l}7 / 8 \\
(87.5 \%)\end{array}$ & $7 / 8(87.5 \%)$ \\
\hline & & Alopecia $>50-\leq 90 \%$ of the body & $\begin{array}{l}1 / 8 \\
(12.5 \%)\end{array}$ & $1 / 8(12.5 \%)$ \\
& & & $\begin{array}{l}0 / 8 \\
(0.0 \%)\end{array}$ & $0 / 8(0.0 \%)$
\end{tabular}

Hair regrowth after treatment on Days 0,28 and $56^{\star}$ and on Day 84

$28^{*} \quad 1>0$ to $50 \%$ hair regrowth

$2 / 8$

$5 / 8(62.5 \%)$

$(25.0 \%)$

$2>50-\leq 90 \%$ hair regrowth

$4 / 8$

$(50.0 \%)$

$3>90 \%$ improvement

$2 / 8$

$(25.0 \%)$

$56^{*} \quad 1>0$ to $50 \%$

$0 / 7$

$(0.0 \%)$

$2>50-\leq 90 \%$

$2 / 7$

$(28.6 \%)$

$3>90 \%$

$5 / 7$

(71.4\%)

$3 / 8(37.5 \%)$

84

$\begin{array}{llll}1 & >0 \text { to } 50 \% & 0 / 7 & 1 / 8(12.5 \%) \\ & >50-\leq 90 \% & (0.0 \%) & \\ 2 & >0 / 7 & 2 / 8(25.0 \%) \\ & & (0.0 \%) & \\ & & 7 / 7 & 5 / 8(62.5 \%) \\ & & (100.0 \%) & \end{array}$

*Hair regrowth assessments were made before treatments were administered on

Days 28 and 56

\section{Safety}

No treatment-related adverse events were recorded in either group throughout the study period. One dog was removed from the fluralaner-treated group during the study because pregnancy was detected on Day 28 and the study protocol excluded pregnant dogs. Mean body weight of all dogs in both groups increased throughout the study. 


\section{Discussion}

Three monthly treatments with the flavored fluralaner $5.46 \%$ chewable tablets were effective in achieving a parasitological cure in client-owned dogs with generalized demodicosis. No mites were found on any fluralaner-treated dog after the Day 28 assessment, while up to 12 applications with topically applied imidacloprid-moxidectin achieved a parasitological cure in 2 of 8 dogs. These findings are consistent with previous studies demonstrating that longer acting fluralaner treatment eliminated natural $D$. canis infestations and variable efficacy of repeated topical treatments with imidacloprid-moxidectin $[6,13-16]$.

Parasitological cure, based on two negative deep skin scrapings one-month apart, does not guarantee a clinical cure, as shown by one imidacloprid-moxidectin treated dog that continued to exhibit severe clinical signs on Day 84 despite zero mite counts on Days 56 and 84. Equally, a strong clinical response does not necessarily indicate parasitological cure: 5 imidacloprid-moxidectin treated dogs improved from severe to mild clinical signs, despite counts of up to 31 mites continuing through Day 84 (one dog that improved from severe to moderate had a Day 84 count of 65 mites). Even with both clinical and parasitological cure, it is important to remember the complex $D$. canis disease pathogenesis.

Predisposing underlying health conditions including hypothyroidism and neoplasia, or immunosuppressive drug treatment are factors that can precipitate clinical demodicosis in adult dogs [19]. Therefore, relapse could occur in successfully treated dogs with continuing underlying health disorders.

\section{Conclusion}

Three consecutive monthly oral administrations to dogs of a flavored chewable fluralaner $(5.46 \% \mathrm{w} / \mathrm{w})$ tablet at a minimum dose of $10 \mathrm{mg} / \mathrm{kg}$ body weight eliminated $D$. canis mites from dogs diagnosed with generalized demodicosis.

\section{Declarations}

\section{Ethics approval and consent to participate}

This study was conducted in compliance with local and national regulations. All dog owners (or delegates) were required to sign and informed consent form prior to any dog being enrolled into the study.

\section{Consent for publication}

Not applicable.

\section{Availability of data and material}

Data from this study are proprietary and maintained by MSD Animal Health.

\section{Competing interests}


NR and FG are employees of MSD Animal Health.

\section{Funding}

The study was funded by MSD Animal Health.

\section{Authors' contributions}

The study design, protocol and report of the study were prepared by NR, LM and FG. LM and his team at Clinvet were responsible for the animal phase, data collection and statistical calculations. All authors revised and approved the final version.

\section{Acknowledgments}

The authors would like to thank all technical and research staff at Clinvet involved in the conduct of the study and Dr Bill Ryan of Ryan Mitchell Associates LLC for support with preparation of the manuscript.

\section{References}

1. Paradis M. New approaches to the treatment of canine demodicosis. Vet Clin N Am Small. 1999;29:1425-36.

2. Paradis $\mathrm{M}, \mathrm{Page} \mathrm{N}$. Topical (pour-on) ivermectin in the treatment of chronic generalized demodicosis in dogs. Vet Dermatol. 1998;9:55-9.

3. Medleau L, Ristic Z. Treating chronic refractory demodicosis in dogs. Vet Med. 1994;89:775-7.

4. Miller WH, Scott DW, Cayette SM, Buerger RG, Bagladi MS. Clinical efficacy of increased dosages of milbemycin oxime for treatment of generalized demodicosis in adult dogs. J Am Vet Med Assoc. 1995;207:1581-4.

5. Six RH, Becskei C, Mazaleski MM, Fourie JJ, Mahabir SP, Myers MR, et al. Efficacy of sarolaner, a novel oral isoxazoline, against two common mite infestations in dogs: Demodex spp. and Otodectes cynotis. Vet Parasitol. 2016;222:62-6.

6. Petersen I, Chiummo R, Zschiesche E, Karas-Tecza J, Rapti D, Roepke R, Thomas E. A European field assessment of the efficacy of fluralaner $\left(\right.$ Bravecto $^{\circledR}$ ) chewable and spot-on formulations for treatment of dogs with generalized demodicosis. Parasit Vectors. 2020;11:304.

7. Bravecto. Summary of Product Characteristics. European Medicines Agency. https://www.ema.europa.eu/en/medicines/veterinary/EPAR/bravecto. Accessed 24 May 2021.

8. FDA NADA Bravecto ${ }^{\circledR}$ https://animaldrugsatfda.fda.gov/adafda/app/search/public/document/downloadFoi/9329. Accessed 24 May 2021.

9. European Medicines Agency. VICH Topic GL9 (GCP). Guideline on Good Clinical Practices. The European Agency for the Evaluation of Medicinal Products (EMEA/CVMP/VICH/595/98-Final). 2000. 
https://www.ema.europa.eu/en/documents/scientific-guideline/vich-gl9-good-clinical-practices-step7_en.pdf. Accessed 24 May 2021.

10. European Medicines Agency. Guideline on statistical principles for veterinary clinical trials (EMA/CVMP/EWP/81976/2010). 2010. https://www.ema.europa.eu/en/documents/scientificguideline/guideline-statistical-principles-clinical-trials-veterinary-medicinal-productspharmaceuticals_en.pdf. Accessed 24 May 2021.

11. European Medicines Agency. Guideline for the testing and evaluation of the efficacy of antiparasitic substances for the treatment and prevention of tick and flea infestation in dogs and cats. 2016. https://www.ema.europa.eu/en/documents/scientific-guideline/guideline-testing-evaluation-efficacyantiparasitic-substances-treatment-prevention-tick-flea_en-0.pdf. Accessed 24 May 2021.

12. Farrington $\mathrm{CP}$, Manning $\mathrm{G}$. Test statistics and sample size formulae for comparative binomial trials with null hypothesis of non-zero risk difference or non-unity relative risk. Stat Med. 2009;9:1447-54.

13. Fourie JJ, Liebenberg JE, Horak IG, Taenzler J, Heckeroth AR, Frénais R. Efficacy of orally administered fluralaner (Bravecto ${ }^{\mathrm{TM}}$ ) or topically applied imidacloprid/moxidectin (Advocate ${ }^{\circledR}$ ) against generalized demodicosis indogs. Parasit Vectors. 2015;8:187.

14. Fourie JJ, Meyer L, Thomas E. Efficacy of topically administered fluralaner or imidacloprid/moxidectin on dogs with generalised demodicosis. Parasit Vectors. 2019;12:59.

15. Beugnet F, Halos L, Larsen D, de Vos C. Efficacy of oral afoxolaner for the treatment of canine generalised demodicosis. Parasite. 2016;23:14.

16. Becskei C, Cuppens O, Mahabir SP. Efficacy and safety of sarolaner against generalized demodicosis in dogs in European countries: a non-inferiority study. Vet Dermatol. 2018;29:203.

17. Miller V, Griffin C, Campbell K. Canine Demodicosis. Muller \& Kirk's small animal dermatology. 7th ed. St. Louis: Elsevier; 2013.

\section{Figures}




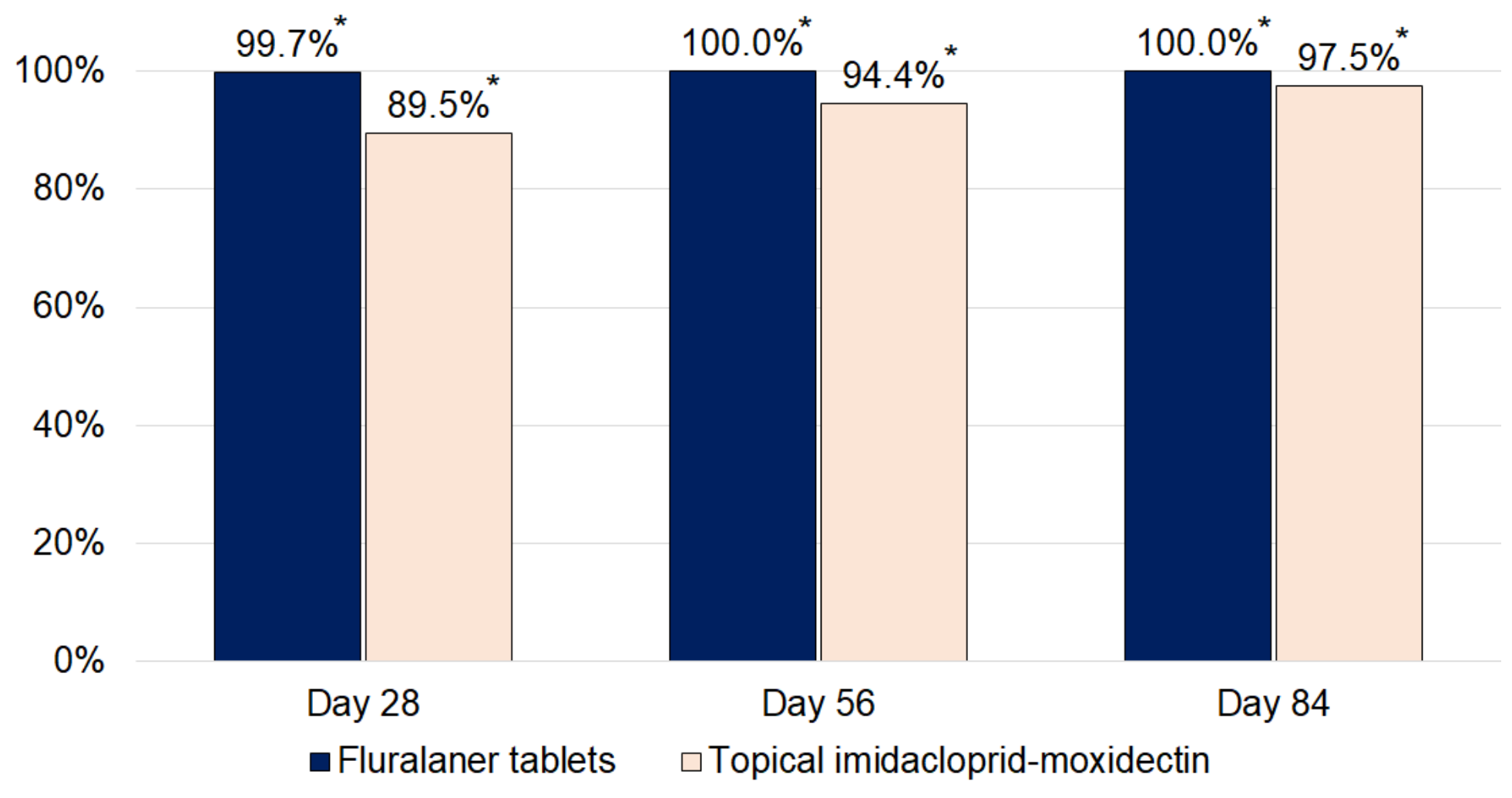

Figure 1

Percentage reduction in Demodex canis mite counts in fluralaner or imidacloprid-moxidectin treated dogs presented with generalized demodicosis

$100 \%$

$100.0 \%$

$100.0 \%$ *

$80 \%$

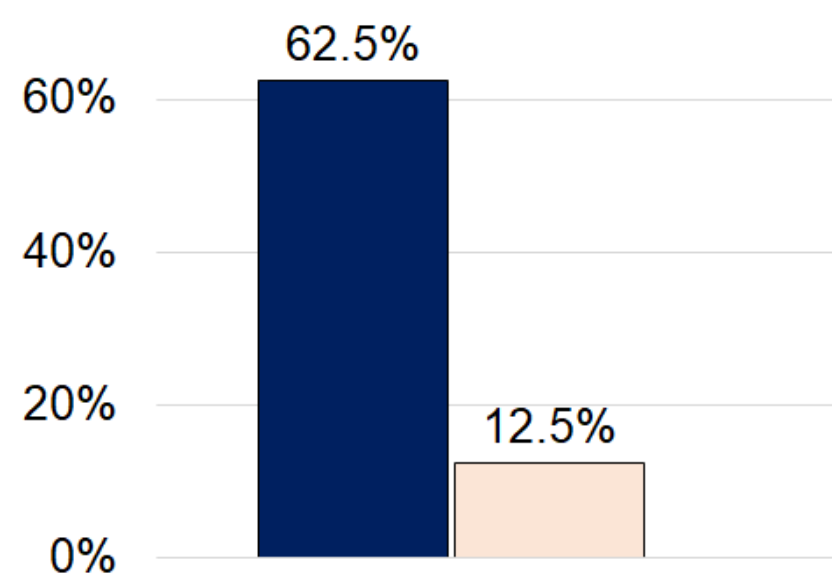

Day 28

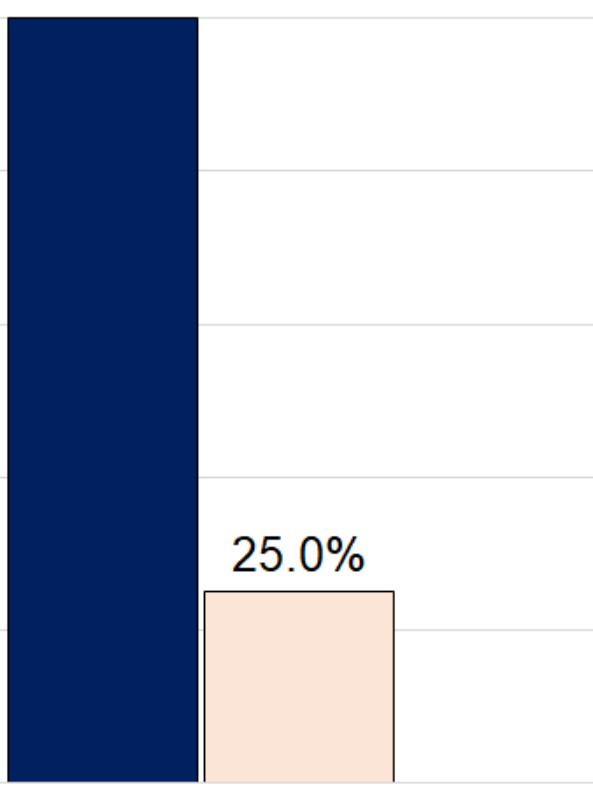

Day 56

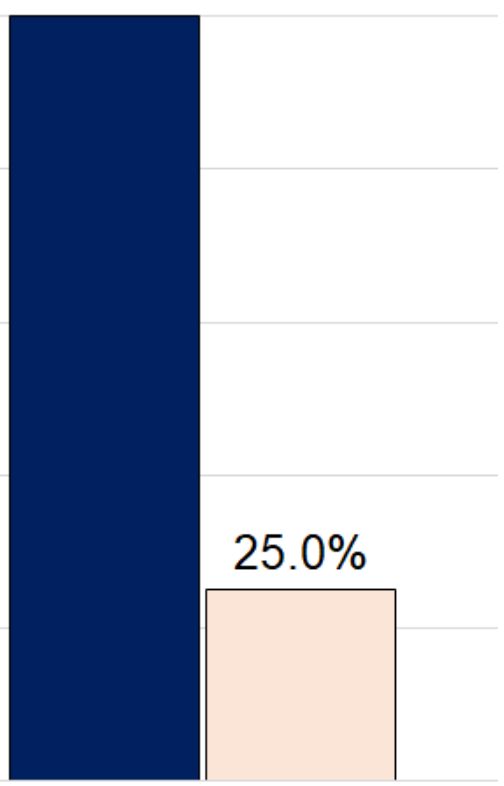

Day 84

- Fluralaner tablets $\quad \square$ Topical imidacloprid-moxidectin 
Figure 2

Percentage of dogs in each group free from Demodex canis mites; *between-group difference significant $(P<0.001)$
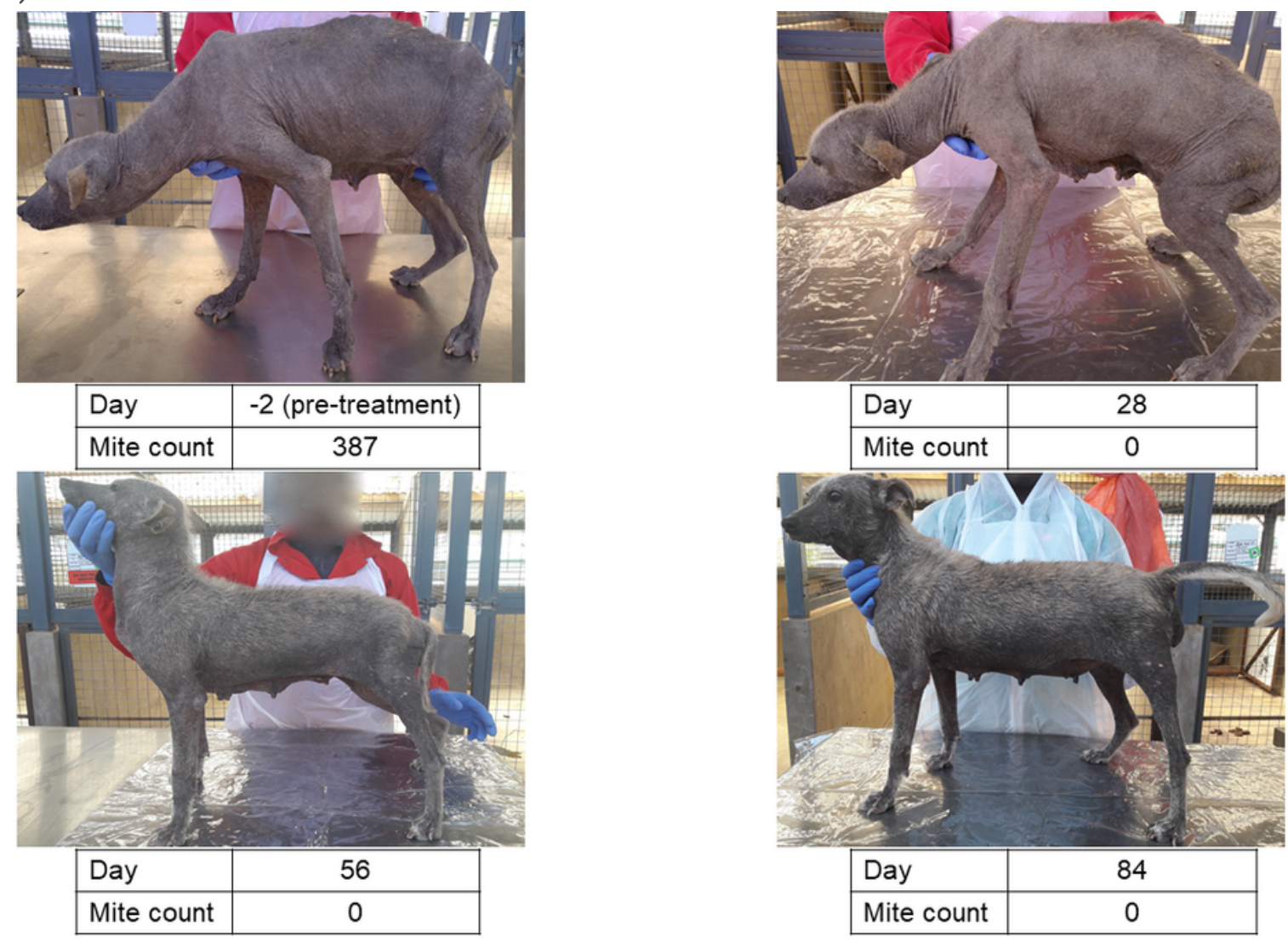

\section{Figure 3}

Dog in the imidacloprid-moxidectin group with a mite count $=0$ on Days 28,56 and 84 but with continuing signs of demodicosis rated as severe 


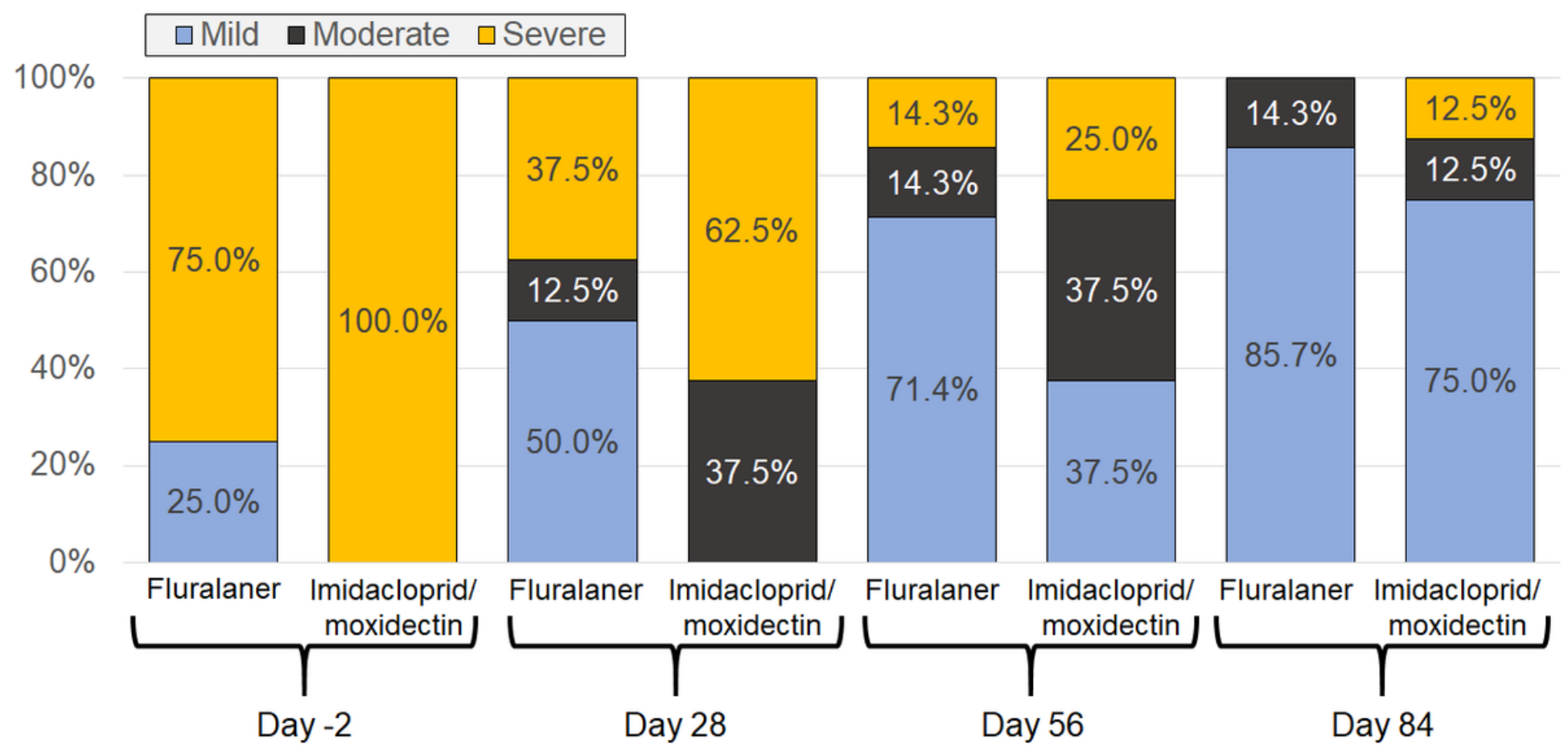

Figure 4

Demodicosis lesion severity in fluralaner or imidacloprid-moxidectin treated dogs on post-treatment Days 28,56 and 84
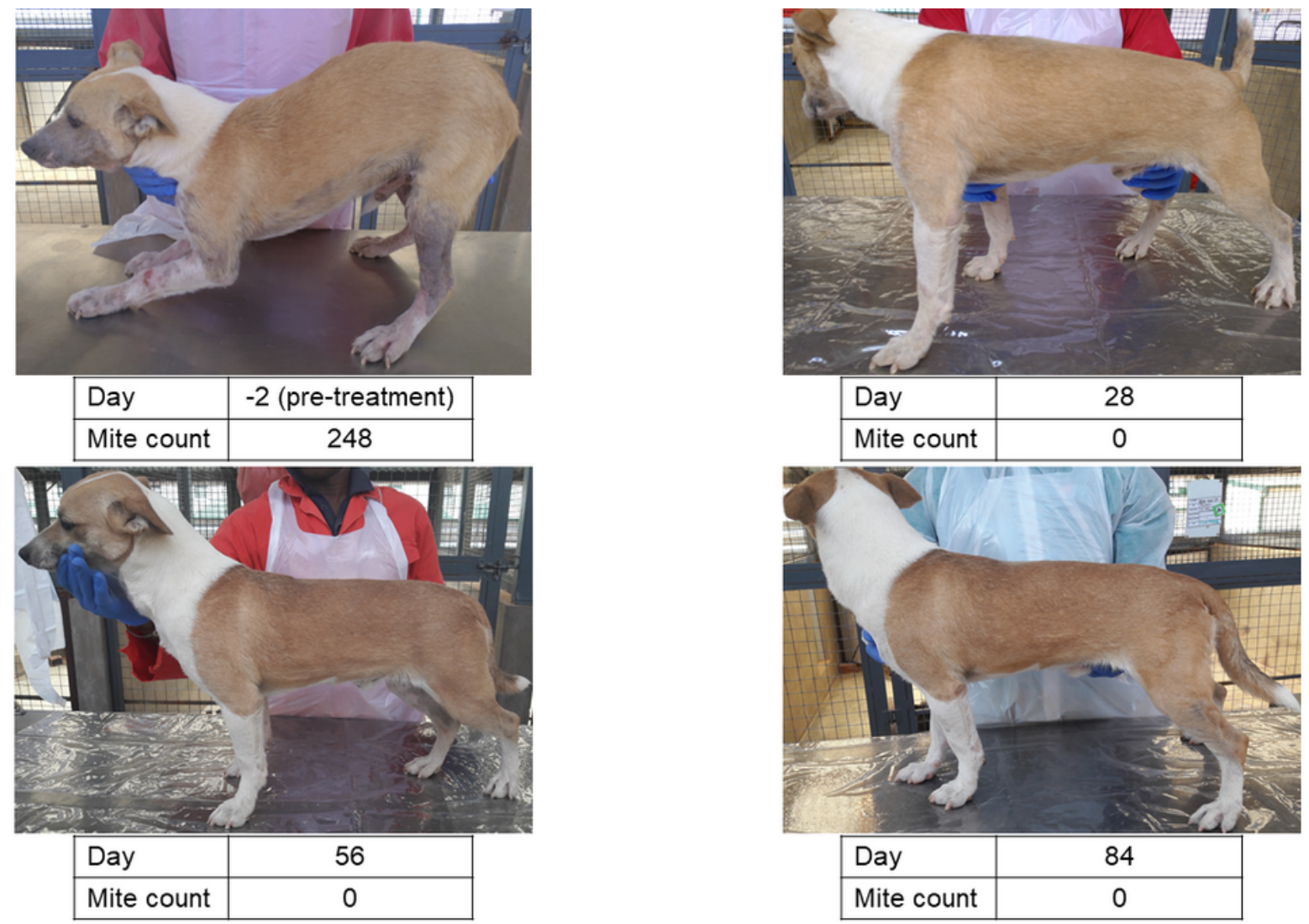

Figure 5 
Pre- and post-treatment picture of dog in the fluralaner group graded as having severe demodicosis lesions on Day -2 